\title{
Tasa de preñez en búfalas sometidas a distintos protocolos de inseminación artificial a tiempo fijo en el nordeste argentino
}

\author{
Crudeli, G.A..; De La Sota, R.L.3; Scarnatto, R.E.'; Konrad, J.L. ; Patiño, E.M. ${ }^{2}$
}

Cátedras de Teriogenología y Tecnología de los Alimentos² ${ }^{1}$ Facultad de Ciencias Veterinarias, UNNE, Sargento Cabral 2139, Corrientes (3400), Argentina. Tel/fax 03783-425753. E-mail: gcrudeli@vet.unne.edu.ar.

${ }^{3}$ Cátedra de Teriogenología, Facultad de Ciencias Veterinarias, Universidad Nacional de La Plata.

\begin{abstract}
Resumen
Crudeli, G.A.; De La Sota, R.L.; Scarnatto, R.E.; Konrad, J.L.; Patiño, E.M.: Tasa de preñez en búfalas sometidas a distintos protocolos de inseminación artificial a tiempo fijo en el nordeste argentino. Rev. vet. 20: 1, 41-44, 2009. El objetivo del trabajo fue comparar las tasas de preñez obtenidas con dos diferentes protocolos de inseminación artificial a tiempo fijo (IATF) en búfalas de raza Mediterránea, en el norte de Corrientes, Argentina. Se utilizaron 93 búfalas pluríparas, con crías, divididas en dos grupos. En el Grupo 1 (G1, n=34) se ensayó el protocolo Ovsynch: sincronización (día 0): 8 ug de buserelina (GnRH); día 7: $150 \mathrm{mg}$ de cloprostenol $\left(\mathrm{PGF}_{2 \alpha}\right)$; día 9: 8 ug de GnRH; día 10: IATF. Resincronización (día 28: 8 ug de GnRH); día 35: diagnóstico de gestación por ultrasonografía y 150 ug de $\mathrm{PGF}_{2 \alpha}$ a los animales diagnosticados vacíos; día 37: 8 ug de GnRH; día 38: IATF. En el Grupo 2 $(\mathrm{G} 2, \mathrm{n}=41)$ se empleó un protocolo con dispositivos intravaginales (DIV) de progestágenos: sincronización (día 0): $2 \mathrm{mg}$ de benzoato de estradiol (BE) y 1 DIV de primer uso durante 7 días; día 7: $150 \mathrm{mg}$ de $\mathrm{PGF}_{2 \alpha}$; día 8: $1 \mathrm{mg}$ de BE; día 9: IATF. Resincronización (día 28): 1 mg de BE y DIV de segundo uso durante 7 días; día 35: diagnóstico de gestación mediante ultrasonografía y 150 ug de $\mathrm{PGF}_{2 \alpha}$ a los animales diagnosticados vacíos; día 36: $1 \mathrm{mg}$ de BE; día 37: IATF. Entre los días 55 y 75 se realizó repaso con toros y el día 100 se efectuó ecografía para determinar preñez final. Los resultados para primo inseminación, resincronización y preñez final en G1 y G2 fueron respectivamente de 44 y $68 \%$ ( $p>0,03$ ), 75 y $80 \%$ y 83 y $87 \%$. Se resalta la más alta tasa de preñez obtenida por el grupo sometido al protocolo con dispositivos intravaginales de progestágenos.
\end{abstract}

Palabras clave: búfalo, inseminación artificial a tiempo fijo, $\mathrm{GnRH}$, estrógeno, progesterona.

\begin{abstract}
Crudeli, G.A.; De La Sota, R.L.; Scarnatto, R.E.; Konrad, J.L.; Patiño, E.M.: Pregnancy rate in buffaloes submitted to different fixed time artificial insemination protocols in Northeast Argentina. Rev. vet. 20: 1, 41-44, 2009. The objective of this trial was to compare the pregnancy rates obtained by two different protocols of fixed time artificial insemination (AIFT) in buffaloes of Mediterranean breed in the north of Corrientes, Argentina. Ninety three pluriparous buffaloes with calves, were used. Animals were divided in two groups. In Group $1(\mathrm{G} 1, \mathrm{n}=34)$ the protocol Ovsynch was rehearsed: synchronization (day 0): busereline 8 ug $(\mathrm{GnRH})$; day 7: cloprostenol $150 \mathrm{mg}\left(\mathrm{PGF}_{2 \alpha}\right)$; day 9: GnRH 8 ug; day 10: AIFT. Resynchronization (day 28: GnRH 8 ug); day 35: diagnostic of gestation by ultrasonography and $\mathrm{PGF}_{2 \alpha} 150$ ug to the animals diagnosed open; day 37: GnRH 8 ug; day 38: AIFT. In the Group $2(\mathrm{G} 2, \mathrm{n}=41)$ a protocol with intravaginal devices (DIV) of progestagens was used: synchronization (day 0): estradiol benzoate (BE) $2 \mathrm{mg}$ and 1 DIV of first use during 7 days; day 7: PGF $_{2 \alpha} 150 \mathrm{mg}$; day 8: BE $1 \mathrm{mg}$; day 9: AIFT. Resynchronization (day 28): BE $1 \mathrm{mg}$ and DIV of second use during 7 days; day 35: diagnostic of gestation by ultrasonography and PGF ${ }_{2 \alpha} 150$ ug to the animals diagnosed open; day 36: BE $1 \mathrm{mg}$; day 37: AIFT. Between days 55 and 75, cleanup bulls were used. On day 100 an echography was made to determine final pregnancy. Results for first insemination, resynchronization, and final pregnancy in G1 and G2 were respectively of 44 and $68 \%$ ( $\mathrm{p}>0.03$ ), 75 and $80 \%$ and 83 and $87 \%$. The highest pregnancy rate obtained by the group submitted to the progestagen intravaginal devices protocol, is emphasized.
\end{abstract}

Key words: buffalo, fixed time artificial insemination, GnRH, oestrogen, progesterone. 


\section{INTRODUCCIÓN}

La inseminación artificial (IA) aún no está suficientemente arraigada en ganado bubalino debido a dificultades en la identificación de las manifestaciones del estro y del momento apropiado para inseminar. Una característica de las búfalas es su baja incidencia $(3,4$ a $16,7 \%$ ) de comportamiento homosexual durante el celo $^{3,12}$, lo que entorpece su visualización externa y obliga a la utilización de retajos para su detección. Tal característica, asociada a variaciones de la duración del estro (6 a 48 horas), torna más trabajosa la detección del celo y dificulta el empleo de la IA ${ }^{5}$. En bovinos, la sincronización del celo y de la ovulación por métodos hormonales ha presentado resultados animadores para el empleo de la IA a tiempo fijo (IATF) ${ }^{16,18}$.

Los protocolos de sincronización existentes permiten realizar IATF (en horario predeterminado) sin necesidad de observar el estro, facilitando el manejo del rodeo y optimizando el empleo a campo de esta biotecnología. El estudio de la dinámica folicular durante el ciclo estral esclarece los fenómenos que interfieren en la sincronización del celo y ovulación. Ella depende del control de algunos factores importantes como la prevención del desarrollo de folículos persistentes que contienen ovocitos envejecidos, la generación de una nueva onda folicular independiente del estado del ciclo estral, la manipulación de la fase luteínica y la sincronización precisa del futuro folículo ovulatorio ${ }^{13}$.

Estudiando la dinámica folicular durante el tratamiento Ovsynch se verificó que después de la primera aplicación de GnRH ocurre la ovulación y/o el inicio de una nueva onda de crecimiento folicular, que resulta en la presencia de un folículo dominante 7 días después, momento en que se aplica $\mathrm{PGF}_{2 \alpha}$. La luteólisis provocada por $\mathrm{PGF}_{2 \alpha}$ hace que todos los animales tratados ovulen 24 a 32 horas después de la segunda dosis de GnRH. Así, Ovsynch revela una gran eficiencia en la sincronización de la ovulación en bovinos ${ }^{16}$.

Otros trabajos demostraron que en búfalas tratadas con este protocolo durante la estación reproductiva, la tasa de concepción resultó 50,2\%, registrándose influencia de la condición corporal en los porcentajes de preñez ${ }^{7}$. En igual sentido, otros autores verificaron interferencia de la condición corporal en la tasa de concepción de búfalas inseminadas artificialmente ${ }^{4,9}$. Para realizar IATF es preciso sincronizar la ovulación de un óvulo fértil en un momento determinado, lo cual se logra induciendo la formación de una nueva onda folicular y su posterior ovulación.

Actualmente existen varios protocolos desarrollados a tal fin, por ejemplo, están los que utilizan GnRH para inducir la generación de una nueva onda, como así también la ovulación al final del protocolo $(O v s y n c h){ }^{16}$. En búfalos de Brasil, tal procedimiento logró 56,5\% de preñez, que ascendió a $64,2 \%$ usando LH en lugar de la segunda dosis de GnRH ${ }^{1}$. En búfalas de raza Mediterránea del norte argentino, en dos años consecutivos
(2002-2003) se obtuvieron tasas de preñez de 50 y $44 \%$ con protocolo Ovsynch ${ }^{14}$.

Existen otros protocolos de IATF que utilizan estrógeno en combinación con progestágenos para lograr esos objetivos. El estrógeno en presencia de progesterona $\left(\mathrm{P}_{4}\right)$ inhibe la liberación de $\mathrm{GnRH}$ y por lo tanto de FSH, induciendo así la atresia de los folículos. Una vez metabolizado el estrógeno se produce la descarga de FSH acumulada, que induce la formación de una nueva onda folicular luego del cuarto día de la aplicación del estrógeno. Al final del protocolo se sincroniza la ovulación nuevamente con estrógeno, ya que éste, en ausencia de $\mathrm{P} 4$ y con un folículo en franca dominancia, induce por feed-back positivo la descarga LH fundamental para la ovulación ${ }^{13}$.

Los dispositivos intravaginales (DIV) con P4 se instalan por 7 a 10 días, simulando la acción del cuerpo lúteo, junto con benzoato de estradiol (BE), el cual genera una nueva onda folicular. Al retirar el dispositivo se aplica $\mathrm{PGF}_{2 \alpha}$ y al día siguiente se coloca nuevamente BE o GnRH para sincronizar la ovulación, inseminándose a las 50-54 horas posteriores al retiro del dispositivo.

En India se utilizaron DIV por ocho días, obteniéndose $80 \%$ de celo al retirar los dispositivos, así como $33 \%$ de preñez a la IATF realizada $60-84$ horas después ${ }^{17}$. En Brasil se utilizó un implante de progestágeno (Crestar) versus un DIV durante 9 días, encontrándose intervalos de 5,5 y 8,7 días respectivamente entre el inicio del tratamiento y la emergencia de la onda, así como tasas de preñez de 47 y $49 \%$ respectivamente 8 . Usando diferentes protocolos de sincronización y resincronización, como ser Ovsynch (G1) comparado con Ovsynch más DIV por 7 días (G2) en la estación reproductiva, versus Ovsynch fuera de la estación reproductiva (G3), a la primo inseminación se obtuvieron porcentajes de preñez de 66,$6 ; 40,5$ y 33,3\% en G1, G2 y G3 respectivamente, en tanto que las tasas de preñez logradas con la resincronización fueron de 33,3; 40,9 y $0 \%$ para los mismos grupos ${ }^{15}$.

En Brasil, utilizando dispositivos de un solo uso versus de segundo uso y evaluando el efecto sobre la onda folicular y la tasa de preñez conseguida, comparando la utilización de hCG o GnRH 48 horas posteriores al retiro del dispositivo, se lograron los siguientes resultados: 1,$39 ; 1,21 ; 1,39$ y $1,11 \mathrm{~mm}$ de diámetro para el folículo dominante, tasas de preñez a la primo inseminación de 49,3; 54,4; 55,6 y 49,1\% respectivamente para hCG, GnRH en dispositivos de primer uso y hCG y GnRH en segundo uso respectivamente ${ }^{11}$.

En Italia, en búfalas lecheras pluríparas de raza Mediterránea, se comparó un dispositivo con P4 (Prid) por 10 días, colocando en un grupo (A) el día 7 PMSG más $\mathrm{PGF}_{2 \alpha}$ y en el (B), el día 10, inseminando a todas ellas 72 horas después del retiro del dispositivo. Los resultados obtenidos fueron de 56,2 y 57,4\% de preñez para los grupos A y B, respectivamente ${ }^{2}$. Algunos sugieren que los altos niveles de progesterona en búfalo condicionan su resultado reproductivo ${ }^{6}$. 
En virtud de la disparidad de resultados obtenidos en hembras bubalinas por otros investigadores, el objetivo del presente trabajo fue comparar la preñez en búfalas sincronizadas con protocolo Ovsynch versus progestágenos.

\section{MATERIAL Y MÉTODOS}

El ensayo fue realizado en la estancia Rincón del Madregon, a $70 \mathrm{~km}$ al sur de la Ciudad de Corrientes, Argentina. Se utilizaron 93 búfalas pluríparas con crías, de raza Mediterránea, con edades de 7 a 11 años y condición corporal promedio de 3,8 evaluada sobre la escala de 1 (emaciada) a 5 (obesa). Las búfalas fueron divididas al azar en dos grupos, a saber:

Grupo $1(\mathrm{G} 1, \mathrm{n}=34)$ protocolo Ovsynch. Sincronización: día 0 con 8 ug de buserelina $\left(\mathrm{GnRH}\right.$, Receptal $^{\mathbb{B}}$, Intervet Argentina); día 7: $150 \mathrm{mg}$ cloprostenol $\left(\mathrm{PGF}_{2 \alpha}\right.$, Preloban $^{\circledR}$, Intervet Argentina); día 9: 8 ug GnRH; día 10: IATF, 16 horas después de la segunda aplicación de GnRH. Resincronización, día 28: 8 ug GnRH; día 35: diagnóstico de gestación mediante ultrasonografía y 150 ug PGF $_{2 \alpha}$ a los animales diagnosticados vacíos; día 37: 8 ug GnRH; día 38: IATF.

Grupo $2(\mathrm{G} 2, \mathrm{n}=41)$ protocolo con progestágenos (DIV). Sincronización: día 0 con $2 \mathrm{mg}$ de BE (benzoato de estradiol ${ }^{\circledR}$, Biogénesis, Argentina) y 1 implante intravaginal de $\mathrm{P}_{4}$ durante 7 días (TRIU-B ${ }^{\circledR}$ de primer uso, Biogénesis, Argentina); día 7: $150 \mathrm{mg}$ de $\mathrm{PGF}_{2 a}$; día 8: $1 \mathrm{mg}$ de BE; día 9: IATF. Resincronización, día 28: $1 \mathrm{mg}$ de BE y 1 TRIU-B ${ }^{\circledR}$ de segundo uso durante 7 días; día 35: diagnóstico de gestación mediante ultrasonografía y 150 ug de $\mathrm{PGF}_{2 \alpha}$ a los animales diagnosticados vacíos; día 36: $1 \mathrm{mg}$ de BE; día 37: IATF.

A partir del día 55 se realizó repaso con toros a los dos grupos, hasta el día 75 , fecha en que se efectuaron ecografías para determinar las hembras que se preñaron en la resincronización. El día 100 se realizó ecografía para determinar preñez por toro y final. Las búfalas fueron inseminadas con semen congelado-descongelado de un búfalo de probada fertilidad. Se utilizó el mismo inseminador para ambos grupos. Los diagnósticos de gestación se realizaron con un ecógrafo PIE MEDICAL ${ }^{\circledR}$. modelo Aquila con transductor rectal de 8 MHz. Los datos de preñez obtenidos para ambas fechas fueron analizados mediante el test de Chi cuadrado, a través del software Statistixversión 1.0, 1999.

\section{RESULTADOS Y DISCUSIÓN}

En la Tabla 1 pueden observarse las tasas de gestación obtenidas para cada tratamiento, a los días 35 y 75 , así como la preñez final. Los datos muestran que los porcentajes de preñez a la primer IATF fueron de 44 y $68 \%$ para G1 y G2 respectivamente, siendo la diferencia significativa $(p<0,03)$. En la resincronización los valores fueron de 75 y $80 \%$ para G1 y G2 respectivamente (diferencia no significativa). Cabe destacar que el escaso número de animales de la segunda IATF torna difícil arribar a conclusiones certeras. La preñez final de 83 y $87 \%$ en sendos grupos, puede ser considerada muy buena para esta especie en nuestra región.

Los resultados obtenidos concuerdan con los relatados por autores brasileros para animales lecheros con nutrición controlada ${ }^{1}$, quienes registraron tasas del $50 \%$ para distintas condiciones corporales. Con relación a trabajos anteriores de nuestro grupo, dicha tasa es inferior a la que obtuvimos con protocolo Ovsynch y superior a la registrada con DIV dentro de la estación reproductiva ${ }^{15}$. Fuera de dicha estación los resultados son más bajos. Los datos de resincronización fueron buenos para ambos tratamientos, aunque el número de animales remanentes fue escaso para inferir estadísticas válidas.

Es importante destacar que las ecografías permitieron detectar varias hembras preñadas en la primera IATF, que luego aparecieron vacías. En G1 y G2 la mortalidad embrionaria (13 y $11 \%$ respectivamente) debería ser cuidadosamente interpretada pues según investigaciones previas, dentro de los 20 a 40 días de gestación ocurre un $22,9 \%$ de pérdidas embrionarias en las búfalas inseminadas ${ }^{10}$. Dicho porcentaje es inferior al reportado para búfalas del nordeste argentino fuera de la estación reproductiva (en enero), donde hubo un $60 \%$ de muerte embrionaria ${ }^{15}$. Quizás el estrés térmico no sea ajeno a tan alta tasa de pérdida de embriones, circunstancia que debería ser motivo de investigación en la zona.

Se concluye que bajo las condiciones del presente ensayo, en la primo inseminación el protocolo DIV logró mayores tasas de preñez que el protocolo Ovsynch, en tanto que las gestaciones fueron similares en la resincronización y preñez final.

Agradecimientos. Al Señor Hernán Gómez Danuzzo y personal del establecimiento "Rincón del Madregon", por facilitar animales e infraestructura para la realización del trabajo. A la Srta. María José Olazarri, por la traducción del resumen.

\section{REFERENCIAS}

1. Araujo Berber R, Madureira EH, Baruselli PS. 2002. Comparation of two Ovsynch protocols (GnRH versus LH) for fixed timed insemination in buffalo (Bubalus bubalis). Theriogenology 57: 1421-1430. 
2. Barile VL, Terzano GM, Allegrini S, Maschio M, Razzano M, Neglia G, Pacelli C. 2007. Relationship among preovulatory follicle, corpus luteum and progesterone in oestrus synchronized buffaloes. J Anim Sci 6: 663-666.

3. Baruselli PS. 1994. Basic requirements for artificial insemination and embryo transfer in buffaloes. Buffalo $J 2$ : $53-60$.

4. Baruselli PS, Barnabe VH, Barnabe RC. 1995. Efeito da condição corporal sob a tasa de gestación en búfalos. Anais Reunião Anual da Sociedade Brasileira de Transferência de Embriões (Atibada, Brasil), p. 229.

5. Baruselli PS, Muccelolo RG, Viana WG, Castro-Junior FG, Reichert RH, Alvarez RH. 1996. Involución uterina no período pós-parto en hembras bubalinas (Bubalus bubalis). Indústr Anim 53: 51-55.

6. Baruselli PS, Mucciolo RG, Visintin JA, Viana WG, Arruda RP, Madureira EH, Oliveiras CA, MoleroFilhof JR. 1997. Ovarian follicular dynamics during the estrous cycle in buffalo (Bubalus bubalis). Theriogenology 47: 1531-1547.

7. Baruselli PS, Oliveira JF, Mattos JC. 1998. Eficiência reprodutiva de búfalos criados no Vale do Ribeira, SP. Anais Congresso Brasileiro de Reprodução Animal (Belo Horizonte, Brasil), p. 285.

8. Baruselli PS, Carvalho NA, Enriquez CH, Nichi M. 2000. Pre-synchronization with GnRH 7 days before Ovsynch protocol for timed insemination in buffalo. Anais I Simpósio de Búfalos de las Américas (Belem, Brasil), p. 414-417.

9. Bhalaru SS, Tiwana MS, Singh N. 1987. Effect of body condition at calving on subsequent reproductive performance in buffaloes. Indian J Anim Sci 57: 33-36.

10. Campanile G, Di Palo R, Neglia G, Vecchio D, Gasparrini B, Prandi A, Galiero G, D'Occhio MJ. 2007. Corpus luteum function and embryonic mortality in buffaloes treated with a $\mathrm{GnRH}$ agonist, hCG and progesterone. Theriogenology 67: 1393-1398.
11. Carvalho NA, Nagasaku EM, Vannucci FS, Toledo LM, Baruselli PS. 2007. Ovulation and conception rates according intravaginal progesterone device and hCG or GnRH to induce ovulation in buffalo during the off breeding season. Ital J Anim Sci 6: 646-648.

12. Crudeli GA, Maldonado Vargas P, Flores Barbarán MS. 1996. Comportamiento reproductivo del búfalo en el nordeste argentino. Actas XV Congreso Panamericano de Ciencias Veterinarias (Campo Grande, Brasil), p. 388.

13. Driancourt MA. 2000. Regulation of ovarian folicular dynamics in faro animals. Implications for manipulation of reproduction. Theriogenology 55: 1211-1239.

14. Kizur A, Pellerano GS, Maldonado Vargas P, Rodríguez S, Crudeli GA. 2003. Eficiencia en el uso del protocolo de sincronización Ovsynch con resincronización en búfalos en el NEA. Anales Reunión de Comunicaciones Cientificas y Tecnológicas de la UNNE (Corrientes, Argentina), $\mathrm{V}-041$.

15. Pellerano G, Crudeli G, Da Silva A, Amuchastegui F. 2005. Evaluación de diferentes protocolos de sincronización y resincronización con inseminación artificial a tiempo fijo en búfalos en el noreste argentino. Anales XXVI Sesión de Comunicaciones Cientificas, Facultad de Ciencias Veterinarias UNNE (Corrientes, Argentina), p. 70.

16. Pursley JR, Mee MO, Wiltbank MC. 1995. Sinchronization of ovulation in dairy cows using PGF2 and GnRH. Theriogenology 44: 915-923.

17. Rajamahendran R, Thamotharam M. 1983. Effect of progesterone releasing intravaginal device (PRID) on fertility in the post-partum buffalo cow. Anim Reprod Sci 6: 111-118.

18. Thatcher WW, Drost M, Savio JD. 1993. New clinical uses of GnRH and its analogues in cattle. Anim Reprod Sci 33: 27-49. 